\title{
Salud bucal: representaciones sociales en madres gestantes de una población urbana. Medellín, Colombia
}

\author{
Oral health: social representations among pregnant mothers. \\ Medellin, Colombia.
}

Gloria Escobar-Paucar ${ }^{1}$

Catalina Sosa-Palacio ${ }^{1}$

Ángela Sánchez-Mejía ${ }^{1}$
${ }^{1}$ Facultad de Odontología, Universidad de Antioquia. Calle 64 No 52-59.

Medellin Colombia. gescobarp@gmail.com gescobarp@ odontologia.udea.edu.co

\begin{abstract}
Based on the theory of social representations, a qualitative investigation was conducted in order to assess social representations in oral heath in pregnant mothers living in an urban environment. Twenty-eight pregnant adult women attending a prenatal program at a health institution in the city of Medellin, Colombia, were interviewed. The interviews were recorded and transcribed; analysis was performed through open, axial and selective coding, in line with grounded theory. Findings revealed that although pregnant mothers are not greatly concerned about oral health after birth and in early childhood, it increases in importance during growth and development of the child when, besides chewing and feeding aspects, it acquires a socially important role. Analysis revealed how social representations anchored in tradition, with new elements from health professional discourses as well as mass media influences coexist in mothers in an urban environment. These include the close relationship between oral health and teeth, health linked to healthy practices as well as recourse to health services and oral health as a social advantage, related to esthetic aspects.
\end{abstract}

Key words Social representations, Oral health, Mothers, Pregnancy, Qualitative Investigation
Resumen A partir de la teoría de las representaciones sociales, se realizó una investigación cualitativa con el propósito de comprender las representaciones sociales del proceso salud-enfermedad bucal en madres gestantes de una población urbana. Se entrevistaron 28 mujeres adultas asistentes al programa prenatal en una institución de salud de la ciudad de Medellín. Las entrevistas fueron grabadas y transcritas y se analizaron mediante codificación abierta, axial y selectiva, de acuerdo con la teoría fundada. Los hallazgos revelaron que si bien para las madres gestantes la boca del hijo no ocupa un lugar preponderante al inicio del ciclo vital, gana importancia con el proceso de crecimiento y desarrollo del niño, cuando además de su papel en la masticación y alimentación, adquiere una carga social relevante. El análisis dio cuenta de cómo confluyen en las madres de una población urbana representaciones arraigadas en la tradición, con nuevas visiones en cuya construcción hay elementos de los discursos profesionales y de los medios de comunicación; entre ellas, la estrecha relación salud bucal-dientes, la salud atada a prácticas saludables y a la utilización de servicios de salud y salud bucal como ventaja social, relacionada con la estética.

Palabras clave Representaciones sociales, Salud bucal, Madres, Gestación, Investigación cualitativa 


\section{Introducción}

La familia juega un papel fundamental como promotora de la salud, pero es en especial a la madre - en su rol de cuidadora - a quien se atribuye mayor responsabilidad por la salud de los niños, responsabilidad que incluye también la salud bucal $^{1-5}$. Sin embargo, el acompañamiento que se hace desde los programas de salud genera con frecuencia resultados paradójicos; muchos de ellos se centran en la transmisión vertical de conocimientos o en estrategias propias de los modelos de la psicología comportamental ${ }^{6-9}$. Esto lleva a un distanciamiento entre las necesidades de las comunidades, su cultura y los mensajes de los profesionales de la salud ${ }^{10}$.

Cardoso e Gomes ${ }^{11}$ señalan que los grupos sociales experimentan concepciones diversas acerca de la enfermedad - en una lógica diferente del saber médico - y que para interpretar los fenómenos corporales las personas se apoyan en nociones, símbolos y sistemas de referencias interiorizadas de acuerdo con sus medios sociales y culturales ${ }^{11}$; así mismo, los interrogantes respecto al sentido de la salud y enfermedad no se reducen a informaciones médicas. De allí que en salud se haya abierto un campo de estudio a partir de la teoría de las represtaciones sociales formulada por Moscovici, entendidas éstas como "sistemas cognitivos que poseen una lógica y un lenguaje propios, lo cual las convierte en 'teorías' o 'ramas del conocimiento' sui generis destinadas a descubrir la realidad y ordenarla"; Son entonces formas de conocimiento socialmente elaboradas y compartidas, más o menos complejas, en las cuales tiene lugar un proceso de elaboración cognitiva y simbólica que orientará los comportamientos ${ }^{12,13}$.

Es con las representaciones, con su naturaleza y sus límites, con quienes deben relacionarse las disciplinas de la salud para realizar su trabajo de prevención, tratamiento y curación; por tanto se ha planteado que la teoría de las representaciones sociales puede contribuir a que las prácticas de salud abandonen la visión de la educación en salud como una simple transmisión de información y la retomen como un proceso complejo de transformaciones en la cotidianidad del encuentro trabajador de la salud-enfermo, del encuentro entre la ciencia y el sentido común ${ }^{14}$, por cuanto reconocerlas establece condiciones favorables a la superación del carecer meramente instrumental de a educación en salud cuando esta se apoya exclusivamente en el saber científico?.

La salud bucal - y en particular la Salud Bucal Colectiva - no ha sido ajena estas reflexiones y ha resaltado la necesidad de considerar aspectos de naturaleza sociocultural y antropológica, que contribuyan a superar el carácter netamente técnico de la odontología, carácter que según Kovaleski et al. se remonta a los orígenes de la misma y constituye una debilidad conceptual e interpretativa, con consecuencias para las prácticas de promoción de la salud ${ }^{15}$. Un número creciente de investigaciones, en particular en Brasil, buscan reconocer las representaciones sociales en diferentes comunidades en relación con la salud-enfermedad bucal, algunas de ellas en cuidadores o madres.

En Colombia, Nieva et al. estudiaron el proceso salud-enfermedad oral en una población urbana marginal y encontraron que las representaciones sociales se construyen, reconstruyen $\mathrm{y}$ transmiten generacionalmente, percibiendo en este proceso un intercambio entre el conocimiento que tienen los trabajadores de la salud y el saber popular; así, la cultura, la tradición, la fe religiosa, la estética, los elementos emotivos y los no racionales determinan las actitudes frente a la cotidianidad y el abordaje del proceso salud-enfermedad ${ }^{16}$. Pero la maternidad, y en particular la gestación, constituye una vivencia generadora de experiencias y cambios tanto físicos como psíquicos que preparan a la mujer para un período único en su vida ${ }^{17-19}$. Esta vivencia debería articularse desde la promoción de la salud y los programas enfocados a la salud bucal del niño, pero no ha sido suficientemente explorada en relación con la salud bucal desde las posibilidades de la investigación cualitativa.

Con el propósito de comprender las representaciones sociales del proceso salud-enfermedad bucal en madres gestantes en relación con la salud bucal de sus futuros hijos, se realizó una investigación cualitativa en madres de un medio urbano. En el presente artículo se desarrolla uno de los fenómenos centrales encontrados, fenómeno relacionado con la importancia que para las madres adquiere la salud bucal de sus hijos a medida que avanza el ciclo vital.

\section{Métodos}

Se realizó un abordaje cualitativo, que partió de reconocer la subjetividad de las realidades y su relación con el contexto ${ }^{20}$, utilizando la teoría fundada desarrollada por Glasser \& Strauss ${ }^{21} y$ Strauss \& Corbin ${ }^{22}$. El protocolo de estudio fue aprobado por el Comité de Ética de la Facultad de Odontología de la Universidad de Antioquia, conforme a las normas internacionales. 
En concordancia con el carácter inductivo de este tipo de pesquisas, no se comenzó con una teoría que probar sino con un área de estudio ${ }^{23}$ en un diseño flexible, en el cual cada hallazgo se convierte en el punto de partida de un nuevo ciclo investigativo dentro del mismo proceso. Con base en los objetivos del proyecto se elaboró un guía de entrevista semi-estructurada y se realizaron tres entrevistas a modo de estudio exploratorio, que permitieron refinar la guía y mejorar las aptitudes de los entrevistadores ${ }^{24}$. Inicialmente las preguntas se orientaron hacia temas relacionados con las expectativas frente al futuro hijo, la boca y su relación con salud, la boca y sus enfermedades, la boca de los niños y el cuidado de la boca; a medida que en el análisis emergieron otros temas, se modificó la guía hacia aquellos aspectos que permitieron explicar los fenómenos encontrados, como la boca y sus aspectos biológicos y sociales, la responsabilidad, el cuidado y el papel de los odontólogos.

Participaron en total veintiocho mujeres gestantes mayores de edad, asistentes al programa de gestantes la institución y residentes en el área urbana de la ciudad de Medellín, Colombia. Para la selección de las participantes se eligió una institución de salud ubicada en el centro de la ciudad, la cual atiende usuarios adscritos al Sistema General de Seguridad Social en Salud y cuenta con un programa educativo prenatal. Las madres fueron invitadas a hacer parte de la investigación de manera voluntaria; cada una de ellas firmó un consentimiento informado y dio su autorización para la grabación de la entrevistas. Para mantener el acuerdo de confidencialidad, las madres se clasificaron de acuerdo con el número de la entrevista en la cual participaron, y los testimonios, se presentan con el número del archivo, la página y las iniciales asignadas a las participantes.

Todas las entrevistas fueron grabadas y transcritas. Los textos resultantes constituyeron la base para el análisis, el cual se orientó hacia la forma más productiva de transformar e interpretar los datos cualitativos a fin de captar las complejidades de los fenómenos sociales que se buscaba comprender ${ }^{25}$. Inicialmente, cada integrante del equipo investigativo realizo un análisis "línea por línea" de los datos; las preguntas, interpretaciones y comparaciones fueron puestos en común y discutidos en reuniones periódicas del equipo, de las cuales surgieron los memos analítico. De este proceso surgieron las categorías incipientes y sus relaciones, a partir de las cuales se hicieron ajustes a la guía de entrevista para reorientar de manera permanente el proceso investigativo, lo que
Lincoln y Guba denominan "reflexividad". Se realizaron en total cuatro rondas de entrevistas y de manera simultánea se avanzó en el análisis siguiendo los momentos que plantea la teoría fundada: codificación abierta, axial, y selectiva ${ }^{22}$ hasta que se obtuvo una estructura más refinada de categorías y relaciones y se logró su saturación e integración en tres fenómenos, con la ayuda de la revisión teórica.

\section{Resultados y discusión}

Si bien para las madres entrevistadas la salud bucal no ocupa un lugar preponderante en el inicio del ciclo vital, a tal punto que no la mencionaron como parte de sus preocupaciones durante la gestación - aspecto descrito en relación con el primer fenómeno en otro trabajo - cuando los investigadores indagaron de manera específica por el tema, se encontró que a medida que avanza el proceso de crecimiento y desarrollo del niño y aparecen los dientes, la boca gana importancia, y además de su papel en la masticación y la alimentación, adquiere una carga social relevante:

"Me parece un poquito inoficioso, de pronto si uno tiene tiempo se pone a cepillarlos, pero un bebé sin dientecitos (...)”. (E8, P11.)

"Yo no, de pronto me preocupa mucho cuando ya le empiecen a nacer los dientes, que los pone muy indispuestos (...) hasta el momento he pensado en eso". (E7, P5.)

La estrecha asociación boca-dientes no es exclusiva de las madres gestantes; hace parte de las representaciones de la población general e incluso es compartida por otros grupos sociales. De acuerdo con Nieva et al. y Abreu et al, "boca sana" genera respuestas relacionadas con ausencia de caries dental o depósitos sobre los dientes y con ausencia de dolor de tipo dental, así como con características propias de los dientes; de otro lado, como la salud y la enfermedad bucal no están asociadas con la muerte, se minimiza su importancia y la boca solamente es relevante cuando por alguna enfermedad genera incapacidad y a causa de ello las personas pierden la única forma de supervivencia, que es el trabajo a través de un cuerpo sano ${ }^{3,16}$.

Sin embargo, esta visión también refleja la influencia de los discursos reduccionistas de la formación odontológica tradicional, y de las representaciones de los propios odontólogos. Los hallazgos de Nieva et al. señalan que los profesionales están condicionados en gran medida por el pensamiento racional transmitido por la acade- 
mia, pero éste no es puro y está también mediado por las representaciones construidas en su grupo social; ejemplo de ello es que consideran que el paciente solamente está enfermo cuando llega con complicaciones propias de problemas dentales ${ }^{16}$. La arcada dentaria, no la boca en tanto ingestora de alimentos, emisora de sonidos, de transmisión verbal de los símbolos, constituye para los profesionales el objeto de la odontología ${ }^{26}$.

Para las madres entrevistadas, la boca tiene connotaciones como órgano que alberga los dientes y por tanto permite realizar la función masticatoria, relacionada con la alimentación:

"Por la salud, por la boca entran los alimentos”. (E9, P3. LMA)

“(...) ni siquiera podía comer porque no tenía dientes”. (E1, P10. O)

La estrecha relación entre salud general y alimentación es señalada por varios Dias et al. y Abreu et al. en comunidades rurales, por cuanto el cuerpo es medio que permite conseguir el sustento para su familia y requiere una buena alimentación ${ }^{3,4}$. Boca y masticación se relacionan de esta manera con la salud general, pero además las madres consideran la alimentación como causa de una buena o mala salud bucal y clasifican los alimentos como "fuertes" o "débiles" según su efecto en la dentición ${ }^{27}$, un aspecto también expresado por las madres entrevistadas:

"Como dicen que la leche fortalece los huesos, lo mismo pasa con los dientes, los alimentos entran a fortalecer" (E8, P7. J.C)

“(...) Personas que no tienen una buena alimentación o balanceada o que coma como cantidad de calcio suficiente o no sé qué otros alimentos, no van a tener la fortaleza para los dientes entonces se le pueden caer más fácilmente". (E1, P9. O)

Al referirse a boca sana, surgen múltiples alusiones a los dientes, producto de la relación ya planteada, pero también a otras enfermedades y condiciones específicas más allá de la caries y la pérdida dentaria, lo cual refleja el contacto con los servicios de salud y la influencia de los medios de comunicación:

"Que al momento de salirle los dientes le salgan bien, blanquitos. Y no solo la boca sino como que todo esto de aquí, que la mandíbula si sea proporcional”. (E1,P7. O)

La gingivitis, el mal aliento, el sarro, la caries, que eso lo ve uno normal en la televisión”. (E1,P9. O)

Otras respuestas evidencian una representación de salud bucal como una serie de cuidados para evitar enfermedades:
"Una boca sana es como visitar también al odontólogo”. (E7, P7. L.A)

"Las personas se enferman de la boca por falta de higiene, por no cepillarse los dientes ni usar la seda”. (E7, P7. J.B)

Este último hallazgo coincide con los de Alzate et al..$^{28}$ y Abreu et al. ${ }^{3}$ y refleja, además de aspectos culturales que relacionan salud con ausencia de enfermedad, un discurso del personal de salud con el cual tienen contacto a través de programas y campañas, donde se atribuyen explicaciones unicausales de tipo biológico a las enfermedades bucales, así como responsabilidades individuales relacionadas con "hábitos poco

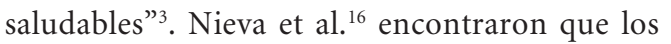
habitantes de una población urbano-marginal clasificaron las causas de enfermedades bucales en endógenas, cuando el individuo tiene pocas posibilidades de intervenir (herencia, desnutrición, condiciones fisiológicas) y exógenas (drogas, hábitos, dieta, trauma, elementos ácidos). Por su parte, los profesionales se centraron en aspectos como la presencia de microorganismos, desnutrición o malos hábitos de higiene ${ }^{23}$.

Surge también la salud-enfermedad bucal como responsabilidad del individuo que sigue o se aparta de las normas y las recomendaciones que se hacen desde el sector salud, no como un estado, un bien en sí mismo o una necesidad ${ }^{3}$. Se minimiza así la relación con determinantes sociales y económicos que explican en gran medida las diferencias en salud bucal ${ }^{29}$ y el hecho de que ésta es resultado de procesos sociales complejos, y no solamente de las prácticas de salud, entre ellas la práctica odontológicai ${ }^{30}$. Con ello, se da paso a la aceptación de la enfermedad como destino ineludible - que llega incluso a hacer parte de la cotidianidad de ciertas comunidades- o a la culpabilización del individuo ${ }^{3,31}$. La boca 'tecnificada', fragmentada y mal comprendida, es vigilada, como lo es el resto del cuerpo, en lo que Kovaleski et al. denominan "disciplinarizacion de la boca"15.

Pero más allá de un órgano con funciones en la masticación y la alimentación, para las madres la boca también representa otros aspectos que superan lo biológico y tocan con lo social, lo afectivo, lo sexual, la comunicación y la estética. Se observa la construcción de una representación que rescata las ventajas que desde el punto de vista social tiene una buena salud bucal. La boca concebida como territorio, donde se configuran los trabajos bucales: masticación (consumo del mundo), lenguaje (producción y consumo de palabras) y erotismo (relación amorosa y producción de actos sexuales), como lo plantea Bo- 
tazzo en su propuesta de la bucalidad ${ }^{32}$. Esta representación toma distancia de la boca vista exclusivamente como un órgano, producto de la fragmentación cartesiana del cuerpo, que conduce a la separación entre el enfermo y su enfermedad, con lo cual se instaura una práctica clínica $\sin$ sujeto $^{32,33}$.

De esta manera, además de hacer parte de la salud general y del cuidado, la salud bucal puesta en el plano de lo social representa un estatus cuya posesión bien puede brindar éxito o fracaso en la vida. De esta manera, se convierte en un medio para ser reconocidos y obtener no solo prestigio social, sino también para tener un lugar en el mundo:

"Porque uno cuando ve a una persona (...) que los dientes torcidos, que no tienen buen aspecto, amarillos cualquier cosa, personalmente a mí me deja mucho que desear”. (E8, P6. G)

"Es muy importante, la apariencia, lo que muestra uno a las demás personas, uno acercársele a alguien con mal aliento(...) uno sabe que eso es lo que está reflejando a las demás personas, el aseo que tengas (...)" (E1,P5. O)

Pero a la vez el cuidado y el estado de los dientes de los niños y niñas reflejan no solamente el estilo de vida de la persona, sino también sus condiciones de vida y dificultades:

"Hay unos niños con los dientes podriditos, los de leche, y uno lo relaciona con personas muy humildes". (E8, P4. G)

Esta dimensión social de la salud bucal está estrechamente relacionada con lo estético, un hallazgo común en los trabajos con diferentes poblaciones $^{3,28}$. Se trata entonces de la salud bucal relacionada con lo grato a la vista, lo bello, lo limpio, lo armónico, lo de olor agradable, más que con la función ${ }^{16}$. En una estrecha relación con la estética, están las emociones que la salud bucal - o su ausencia - despiertan; se perciben sentimientos de satisfacción frente a una boca bonita o unos dientes blancos, y sentimientos de molestia, asco o repudio frente a una boca con mal aliento, descuidada o sin dientes ${ }^{1}$. También Abreu et al. reportaron que la pérdida de un diente afecta la sonrisa y hace que las personas sean vulnerables a sentimientos de inferioridad, rechazo e inseguridad ${ }^{3}$, un aspecto también señalado por las madres entrevistadas:

"Yo como me voy a sonreír con usted con un diente mueco, yo me sentiría tenaz (...)”. (E5, P10. Y)

"Es desagradable y uno lo primero que se imagina es que es desaseada, que le vea los dientes feos, disparejos (...)”. (E2, P8-9. C.A)
El grupo otorga al ser humano características que considera como oportunas, tanto desde el punto de vista físico como intelectual y moral, y la familia juega un papel importante en la construcción de esos valores ${ }^{3}$. De allí que, para las madres, apartarse de esas características constituye una situación desfavorable que puede generar discriminación:

"La sociedad discrimina por la apariencia física, incluyendo los dientes. A veces uno estigmatiza mucho a la gente, el mero hecho de tener una cosita en lo físico, ya empieza uno a dejarlos a un lado $(. .$.$) es una sociedad que a veces estigmatiza$ a la gente, que la pone allá afuerita (...) quizás ya la queramos después cuando tenga los dientes bonitos (...) es normal que suceda”. (E6, P8. O)

De esta manera, si buena salud bucal se convierte en una ventaja social, a las madres les preocupa que la mala salud bucal de sus hijos los haga objeto de sentimientos negativos y discriminación y los afecte en su futuro. Tal preocupación se refleja en sentimientos de responsabilidad y culpa:

“(...) entonces no crearle un complejo (al hijo) por algún defecto en los dientes”. (E1, P4. O)

"Si uno les enseña a cuidarse los dientes, esos rechazos no los van a afectar". (E.10. P4. E.P)

Vale la pena resaltar que la relación entre estética y ventaja social es propia de transformaciones en el ideal del hombre contemporáneo, para quien el culto al cuerpo y a la belleza constituyen valores a ser cultivados socialmente; un cuerpo sano y una sonrisa perfecta se han constituido en el ideal al que se debe sujetar todo ser humano. Como lo plantea Lipovetsky, el gran auge y expansión del culto al cuerpo a partir de los años ochenta, ha generado un mercado considerable alrededor del cuerpo ${ }^{34}$, mercado que se traduce en la práctica del deporte, productos para el cuidado de la belleza y cirugías estéticas, en el afán de arreglar el cuerpo y mejorarlo, y que toca actualmente también a la práctica odontológica. Parte de esta transformación descansa en los medios masivos de comunicación, con la creación de estereotipos frente a la belleza y la boca:

"Actualmente con los medios de comunicación, mira por ejemplo que uno ve todos los presentadores, con los dientes súper lindos, blancos, que rico uno tener los dientes de esa forma". (E1, P8. O)

De otro lado, posicionar la salud bucal como ventaja social, conlleva el riesgo de que su ausencia se convierta en exclusión, como resultado de la organización de los servicios odontológicos. 
De esta manera, el valor no es la salud bucal en sí misma; lo es la intervención, que pasa a convertirse en necesidad, en una inversión en la escala de las necesidades humanas que legitima la práctica odontológica transformada en mercancía, a la cual tienen acceso únicamente aquellas personas que poseen los recursos necesarios ${ }^{35}$.

También surgió entre las madres entrevistadas, aunque con menos fuerza, una representación donde la salud bucal se inscribe en el mundo de lo simbólico: el lenguaje y la comunicación.

"Yo me di cuenta que la parte dental es una parte importante, porque socialmente juega mucho en la comunicación de las personas". (E3, P3. P)

"He pensado en que pueda hablar y comunicarse, y boca sana también es el lenguaje. Es muy importante porque uno constantemente está hablando con las personas". (E3, P6. P)

Es de resaltar que en el presente trabajo aparecieron diferencias entre las representaciones de las madres entrevistadas, pertenecientes a un medio urbano y con acceso a servicios de salud como lo refleja su vinculación a un programa prenatal - y los hallazgos reportados en poblaciones rurales o urbano-marginales, donde la salud bucal es una realidad deseada por las mujeres, en tensión con la realidad vivida de pérdida de dientes ${ }^{3,28}$. Las madres entrevistadas son conscientes de la forma en que han cambiado las representaciones y con ellas la importancia de la boca, a diferencia de lo que ocurría en generaciones anteriores:

"Estamos en otro tiempo, ahora ya hay más conciencia en muchas cosas (...), ellos fueron familias de 20, 15 hijos, entonces los papás no podían estar pendientes, eran pendientes de otras cosas, y no que venga le miro los dientes, que lo llevo a odontología". (E2, P8. C.A)

"En ese tiempo como que no se preocupaban tanto por lo de la boca, en cambio ahora sí (...), y uno se preocupa porque se cepillen desde que nacen". (E9 , P4. N)

Ello refleja, como lo señalan Cardoso y Gomes $^{11}$, diferentes concepciones de salud- enfermedad, entre grupos humanos ${ }^{11}$, pero también que las representaciones sociales son "consensos dinámicos", por cuanto no significan uniformidad ni excluyen la diversidad: es posible que coexistan conceptos e imágenes diferentes, unas de ellas hegemónicas, otras emancipadas y algunas polémicas ${ }^{36}$.

\section{Consideraciones finales}

El presente trabajo muestra cómo confluyen en las madres de una población urbana representaciones acerca del proceso salud-enfermedad bucal, arraigadas en la tradición, con nuevas visiones, en cuya construcción hay elementos de los discursos profesionales y de los medios de comunicación. Es así como la estrecha asociación boca-dientes - reforzada en gran medida por la visión reduccionista y mecanicista de la salud concebida bajo la influencia del paradigma carte$\operatorname{siano}^{37}$ - explica en parte que las madres otorguen menos importancia a la salud bucal al inicio del ciclo vital, pero en la medida en que el niño crece, ésta adquiere un papel relevante desde el punto de vista social.

De otro lado, la salud bucal atada a prácticas saludables y a la utilización de servicios de salud - una visión reproducida desde la práctica tradicional de la odontología y que se incorpora a las representaciones sociales de las madres - abre paso a la 'boca disciplinada' y 'boca controlada' desde el nacimiento ${ }^{15}$, magnifica la responsabilidad de las madres por la salud bucal de sus hijos, e incluso puede contribuir a culpabilizarlas. Se corre así el riesgo de restarles autonomía - por cuanto se considera que la enfermedad es consecuencia de individuos que no saben o no se quieren cuidar ${ }^{35}$. Lo anterior, sumado a la salud bucal como ventaja social - en estrecha relación con la estética - donde la intervención y no la salud se convierte en el valor deseable ${ }^{35}$, crea dependencia de los servicios, un paso hacia la mercantilización del cuidado.

Surge entonces el reto de fortalecer la autonomía de las madres como cuidadoras y a la vez retomar las múltiples dimensiones de salud bucal presentes en sus representaciones, en cuanto la boca, más allá de lo biológico, abre posibilidades al llamado para 'desodontologizar' la salud bucal, presente en los planteamientos de la Salud Bucal Colectiva ${ }^{35,38}$. Es necesario superar la concepción de la boca vista exclusivamente como un órgano, a través de propuestas como la bucali$\mathrm{dad}^{32}$, de tal forma que la salud no se agote en un acto clínico, en una relación objeto-objeto reducida a la utilización de técnicas ${ }^{39}$. Esto es fundamental para lograr una ruptura epistemológica que permita la emergencia del sujeto, tan necesaria para avanzar hacia prácticas de salud donde cuidar sea más que construir un objeto e intervenir sobre él, en la construcción de una actitud 'cuidadora' que oriente las acciones ${ }^{40}$. 


\section{Contribuciones}

G Escobar-Paucar trabajó en la concepción inicial del trabajo, la construcción del referente teóricometodológico que orientó la investigación, la elaboración de las entrevistas, el análisis de la información y la redacción y aprobación del contenido final del artículo. C Sosa-Palacio participó en la construcción del referente teórico-metodológico que orientó la investigación, la elaboración de las entrevistas, el análisis de la información y la redacción y aprobación del contenido final del artículo. A Sánchez-Mejía participó en la construcción del referente teórico-metodológico que orientó la investigación, la elaboración de las entrevistas, el análisis de la información y la redacción y aprobación del contenido final del artículo.

\section{Referencias}

1. Couto CMMM, Rio LMSP, Martins RD, Martins CC, Paiva SM. A percepção de mães pertencentes a diferentes níveis socioeconómicos sobre a saúde bucal dos seus filhos bebés. Arq Odontol 2001; 37(2):121-132.

2. Martin VB, Angelo M. A organização familiar para o cuidado dos filhos: percepção das mães em uma comunidade de baixa renda. Rev Lat Am Enfermagem 1999; 7(4):89-97.

3. Abreu MHNG, Pordeus IA, Modena CM. Representações sociais de saúde bucal entre mães no meio rural de Itaúna (MG), 2002. Cien Saude Colet 2005; 10(1):245-259.

4. Dias G, Frenceschini SCC, Reis JR, Reis RS, Siqueira-Batista R, Cotta RMM. A vida nos olhos, o Coração nas mãos: concepções e representações femininas do processo saúde-doença. Hist Cienc Saude Manguinhos 2007; 14(3):779-800.

5. Reisine S, Douglas JM. Psychosocial and Behavioral Issues in ECC. Community Dent Oral Epidemiol 1998; 26(Supl. 1):32-44.

6. Gómez SS, Weber AA, Emilson C. A Prospective Study of a Caries Prevention Program in Pregnant Women and their Children Five and Six Years of Age. ASDC J Dent Child 2001; 192(3):191-195.

7. Primosch RE, Balsewich CM, Thomas CN. Outcomes Assessment and Intervention Strategy to Improve Parental Compliance to Follow-up Evaluations after Treatment of Early Childhood Caries using General Anesthesia in a Medicaid Population. ASDC J Dent Child 2001; 63(2):102-108.

8. Peñaranda F, Bastidas M, Escobar G, Torres J.N, Arango A. Análisis integral de las prácticas pedagógicas de un programa educativo en Colombia. $\mathrm{Sa}$ lud Publica Mex 2006; 48(3):229-235.

9. Gazzinelli MF. Educação em saúde: conhecimentos, representações sociais e experiências da doença. Cad Saude Publica 2005; 27(1):200-206.

10. Bastidas M, Peñaranda F, Escobar G, Torres N, Arango A. La comprensión de los significados que del programa de crecimiento y desarrollo tienen sus actores: un paso hacia su cualificación. Cien Saude Colet 2009; 14(5):1919-1928.

11. Cardoso MHC, Gomes R. Social Representations and History: Theoretical and Methodological Principles for Public Health. Cad Saude Publica 2000; 16(2):499-506.

12. Farr M. Las Representaciones Sociales. En: Moscovici S. Psicología Social. Barcelona: Paidós; 1988. p. 496-506.

13. Jodelet D. La Representación Social: Fenómenos, Concepto Y Teoría. En: Moscovici S. Psicología Social. Barcelona: Paidós; 1988. p. 507-534.

14. Guimaraes MCTV. Las Representaciones Sociales: Herramientas para el Diagnóstico de Necesidades de Salud. Avances en Enfermería 1997; 15(1-2):115123.

15. Kovaleski DF, Freitas SFT, Botazzo C. Disciplinarizacao da boca, a autonomia do individuo na sociedade do trabalho. Cien Saude Colet 2006; 11(1):97103. 
16. Nieva BC, Jácome S, Cendales L. Representaciones sociales del proceso salud-enfermedad oral en poblaciones urbano-marginales y su relación con los discursos y las prácticas institucionales. Rev Fed Odontol Colom [internet]. 1999 Ene-Mar [acceso 2011 sep 26]; 57(195):9-56. Disponible en: http://www. encolombia.com/representaciones_odonto.htm

17. Montes MJ. Cuerpos gestantes y orden social: Discursos y prácticas en el embarazo. Index Enferm 2008; 17(1):25-29.

18. Stern DN. La constelación maternal. Un enfoque unificado de la psicoterapia con padres e hijos. Barcelona: Paidós; 1997.

19. Brazelton TB, Cramer BG. La relación más temprana. Padres, bebés y el drama del apego inicial. Barcelona: Paidós; 1993.

20. Lincoln Y, Guba E. Paradigmatic controversies, contradictions and emerging confluences. En: Denzin N, Lincoln Y. Handbook of qualitative research. Thousand Oaks: Sage; 2000. p. 163-188.

21. Glasser BG, Strauss Al. The Discovery of Grounded Theory: Strategies for Qualitative Research. Nueva York: Aldyne; 1967.

22. Strauss A, Corbin J. Bases de la investigación cualitativa. Técnicas y procedimientos para desarrollar la Teoría Fundamentada. Medellín: Editorial Universidad de Antioquia; 2002.

23. Pardo de Vélez G. Investigación Cualitativa. En: Pardo de Vélez G. Investigación En Salud. Factores Sociales. Santafé De Bogotá: Mc-Graw Hill- Interamericana; 1997. p. 181-219.

24. De la Cuesta C. Tomarse El Amor en Serio: Contexto del Embarazo en la Adolescencia. Medellín: Universidad de Antioquia; 2002.

25. Coffey A, Atkinson P. Encontrar el sentido a los datos cualitativos. Estrategias complementarias de investigación. Medellín: Universidad de Antioquia; 2003.

26. Iyda M. Saúde bucal: uma prática social. En: Botazzo C, Freitas SFT. Ciências sociais e saúde bucal: questões e perspectivas. Edusc-Unesp, Sao Paulo. 1998. Citado por Figueiredo GO, Brito DTS, Botazzo C. Ideología, fetiche e utopia na salude: uma análise a partir de saúde bucal. Cien Saude Colet 2003; 8(3):753-763.

27. Bardal PA, Olympio KPD, Valle AAL, Tomita NE. Carie dentária em crianças como fenómeno natural ou patológico: ênfase na abordagem qualitativa. Cien Saude Colet 2006; 11(1):161-167.
28. Alzate TM, Campo LF, Martínez CM, Rueda AY, Tobón JD. Representaciones sociales de los adolescente escolarizados sobre la boca y la higiene oral Medellín, 2004. CES Odontol 2005; 18(2):9-18.

29. Antunes JL, Narvai PC, Nugent ZJ. Measuring inequalities in the distribution of dental caries. Community Dent Oral Epidemiol 2004; 32(1):41-48.

30. Narvai PC. Saúde bucal coletiva, bucalidade e antropofagia. Cien Saude Colet 2006; 11(1):18-21.

31. Romero Y. Hábitos que influyen en el proceso salud-enfermedad bucal. Estudio etnográfico e un grupo de madres de la comunidad de San Isidro, Estado Mérida. Acta odontol Venez 2006; 44(1):70-79.

32. Botazzo C. Sobre bucalidade: notas para a pesquisa e contribuição ao debate. Cien Saude Colet 2006: 11(1):7-17.

33. Cortés N. De lo bucal a lo oral. La práctica odontológica: de la técnica al sujeto [tesis]. Medellín: Facultad de Ciencias Sociales y Humanas Universidad de Antioquia; 2000.

34. Lipoverski G. La era del vacío. Barcelona: Anagrama; 1986.

35. Figueiredo GO, Brito DTS, Botazzo C. Ideología, fetiche e utopia na salude: uma análise a partir de saúde bucal. Cien Saude Colet 2003; 8(3):753-763.

36. Moscovici S. Notes towards a description of social representations. Eur J Social Psychol 1988; 18(3):211250. Citado por Rodriguez T. El Debate de las Representaciones sociales. Relac 2003; 24(93):51-80.

37. Ríos ERG, Franchi KMB, Silva RM, Amorim RF, Costa NC. Senso comum, ciência e filosofia - elo dos saberes necessários a promoção da saúde. Cien Saude Colet 2007;12(2):501-507.

38. Narvai PC. Saúde bucal coletiva: caminhos da odontologia sanitária a bucalidade. Rev Saude Publica 2006; 40(N Esp.):141-147.

39. Cortés N. La práctica odontológica y la subjetividad. Rev Fac Odontol Univ Ant 2001; 12(1):70-72.

40. Ayres JRCM. Sujeito, intersubjetividade e práticas de saúde. Cien Saude Colet 2001; 6(1):63-72.

Artigo apresentado em 21/07/2009

Aprovado em 28/01/2010

Versão final apresentada em 15/02/2010 